Commentary/Correspondence on Hoy R.F. and Chambers D.C.

Silica-related diseases in the modern world

Text: 511 words

\title{
Silica-related diseases in the modern world: a role for self-DNA sensing in lung inflammatory diseases
}

Dieudonnée Togbe ${ }^{1}$, Sulayman Benmerzoug ${ }^{2 *}$, Muazzam Jacobs ${ }^{3}$, Bernhard Ryffel $^{1,3}$ and Valerie FJ Quesniaux².

${ }^{1}$ Artimmune SAS, rue Buffon, 45071 Orleans-Cedex 2, France.

${ }^{2}$ Experimental and Molecular Immunology and Neurogenetics, UMR7355, CNRS and University of Orleans, France.

${ }^{3}$ Institute of Infectious Disease and Molecular Medicine, University of Cape Town, South Africa Correspondence: Valerie F.J. Quesniaux, Email: quesniaux@cnrs-orleans.fr

Note: *Present address: Department of Urology, Urology Research Unit, CHUV, Lausanne, Switzerland

Acknowledgements: The authors work was supported by the French-South-African Partenariats Hubert Curien PROTEA 2019 (Project N 42458RC), and by European funding in Region Centre-Val de Loire (FEDER N²016-00110366 and EX005756).

Occupational silica dust exposure induces inflammatory and fibrotic lung disease such as silicosis, often considered as a disease of the past. However, a recent contribution in this journal highlights new causes of silicosis and reviews the clinical patterns of silicosis, which still represents a major concern of pneumoconiosis for man, lacking effective therapeutic treatment beside lung transplantation ${ }^{1}$.

While protective measures could drastically prevent occupational silica exposure, they are insufficiently implemented. Understanding the pathogenesis mechanisms involved in silicosis should help identify novel therapeutic targets. How silica particles cause inflammation and progressive fibrosis is still unresolved, and merits further investigation. The activation of several pathways have been established. The activation of TNF $\alpha$, alarmins, and NLRP3 inflammasome/IL-1 $\beta$ axis following crystalline silica engulfment by alveolar macrophages, reactive oxygen species production and lysosomal damage, are important drivers of inflammation, as reviewed ${ }^{1}$. 
We wish to add a new dimension to this landscape, involving the recently characterized nucleic acid sensing pathways which show that stimulator of interferon genes (STING) is essential for silica-induced lung inflammation. Indeed, exposure to silica particles induces cell stress and cell death in vitro and in vivo, leading to the release of self-DNA of nuclear and mitochondrial origin in the cytosol and extracellular space ${ }^{2}$. This release of self-DNA in turns activates the DNA sensor/STING pathway and drives type I IFN expression and related inflammation ${ }^{2}$. In vitro, mitochondrial dsDNA is sensed by cGAS-STING in dendritic cells, while in macrophages extracellular dsDNA activates STING via other DNA sensors independently of cGAS after silica exposure (Figure). In vivo, cell free dsDNA is detected in the bronchoalveolar fluid and serum upon experimental lung exposure to silica, leading to IFN-I-driven inflammation ${ }^{2}$. This response to silica exposure is prevented in mice deficient for cGAS, STING or type I IFN receptor. Importantly, self-DNA degradation by administration of DNase I attenuates silica-induced lung inflammation in mice ${ }^{2}$.

Silica exposure is a known factor diminishing host resistance to lung infections, especially increasing the risk of pulmonary mycobacterial infection, but the underlying mechanism is unclear. We propose a role for silica-induced DNA sensing activation. Indeed, while host control of mycobacterial infection relies on type 1 immunity, we found that silica exposure reduced host resistance by activating the DNA/cGAS/STING/Type I IFN pathway that in turns enhances type 2 immunity, and favors mycobacterial infection in mice ${ }^{3}$.

In summary, silica exposure induced cell stress/death causes mitochondrial and nuclear selfDNA release which is recognized by the DNA sensors cGAS and STING and triggers lung inflammation. Such pathogenesis based on self-DNA recognition pathways may be involved more broadly in lung inflammation ${ }^{4}$. Indeed, self-DNA release activating the cGAS/STING pathway is involved in several models of inflammatory lung diseases in mice such as exposure to cigarette smoke ${ }^{5}$, allergens ${ }^{6}$, ozone and particles (unpublished), that depend on cGAS and/or STING expression. Both cGAS and STING are druggable and several small molecules which target these respective pathways are currently in pharmaceutical development.

In conclusion, the recent discoveries suggest that targeting self-DNA or DNA sensors such as cGAS/STING needs to be explored and may lead to novel therapies of silicosis and other severe inflammatory lung diseases. 


\section{References}

1. Hoy RF, Chambers DC. Silica-related diseases in the modern world. Allergy 2020.

2. Benmerzoug S, Rose S, Bounab B, et al. STING-dependent sensing of self-DNA drives silicainduced lung inflammation. Nat Commun. 2018; 9(1): 5226.

3. Benmerzoug S, Bounab B, Rose $S$, et al. Sterile lung inflammation induced by silica exacerbates Mycobacterium tuberculosis infection via STING-dependent type 2 immunity Cell Reports. 2019; 27: $2649-2664$.

4. Benmerzoug S, Ryffel B, Togbe D, Quesniaux VFJ. Self-DNA Sensing in Lung Inflammatory Diseases. Trends in immunolog.y 2019; 40(8): 719-734.

5. Nascimento M, Gombault A, Lacerda-Queiroz N, et al. Self-DNA release and STINGdependent sensing drives inflammation to cigarette smoke in mice. Sci Rep. 2019; 9(1): 14848.

6. Han Y, Chen L, Liu H, et al. Airway Epithelial cGAS Is Critical for Induction of Experimental Allergic Airway Inflammation. J Immunol. 2020; 204(6): 1437-1447. 


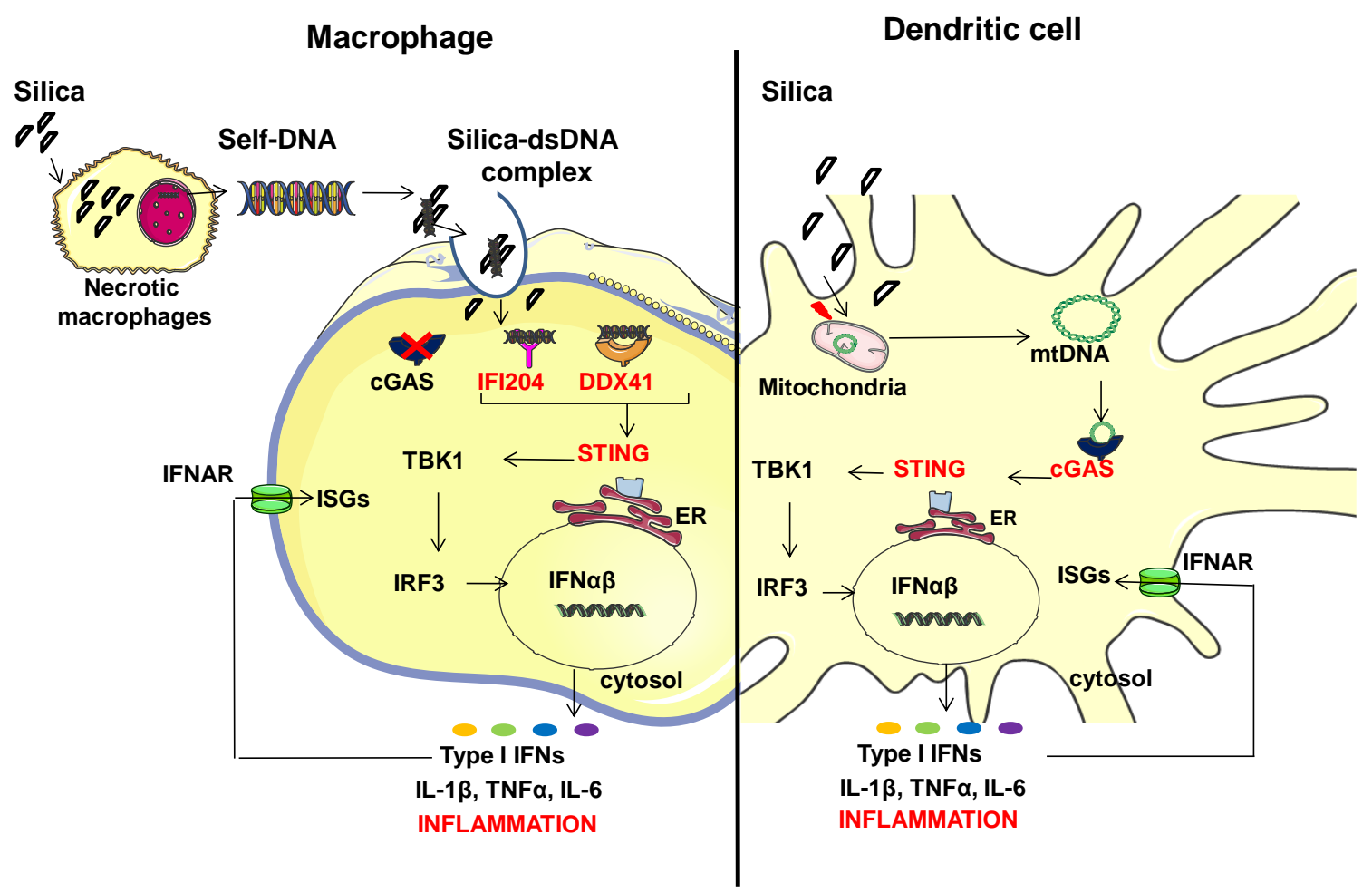

Figure 1: Silica-induced inflammation through DNA sensors / STING / type I Interferon pathway in macrophage and dendritic cell.

Silica microcrystals induce cell stress characterized by mitochondrial damage and release of mitochondrial DNA (mtDNA) and nuclear DNA (nDNA) into the cytosol, and cell death with release of mtDNA and nDNA in the extracellular milieu. Ectopic self-DNA is internalized by macrophages and is sensed by cytosolic sensors that trigger the stimulator of interferon genes (STING) pathway. In vitro silica-exposed macrophages are activated by extracellular DNA, leading to STING-dependent type I IFN response, and this seems independent of cGAS but involving DDX41 and IFI204 DNA sensors. In dendritic cells, silica exposure induced mtDNA engages cGAS to form 2'3'cGAMP and activate STING. After self-DNA recognition, STING recruits the TANK-binding kinase 1 (TBK1), which dimerizes and autophosphorylates; activated TBK1 in turns phosphorylates STING, creating a docking site for the IFN regulatory factor 3 (IRF3); IRF3 is phosphorylated by TBK1, leading to IRF3 dimerization and translocation to the nucleus where it triggers IFN $\alpha$ and IFN $\beta$ gene expression, inducing downstream type I IFN responses with activation of interferon simulated genes (ISG). STING activation also leads to NF-KB activation and the production of pro-inflammatory cytokines. 
